\title{
Aero-optical wavefront measurement technique based on BOS and its applications
}

\author{
TIAN LiFeng ${ }^{*}$, YI ShiHe, ZHAO YuXin, HE Lin \& CHEN Zhi \\ College of Aerospace and Material Engineering, National University of Defense Technology, Changsha 410073, China
}

Received February 17, 2011; accepted April 6, 2011

\begin{abstract}
A collimated light beam will be refracted if it propagates through a flow-field with index-of-refraction variations, and its wavefront will be distorted. If we measure the deflection angle of the light beam, the gradient of the wavefront can be obtained using the Malus law, and the wavefront aberration can be computed with a reconstruction algorithm. Two characteristics of background oriented schlieren (BOS) are conducive to wavefront aberration measurement: BOS can be used to measure the deflection angle of a light beam by measuring the displacement field between the reference image and the experiment image. Moreover, in a BOS system of Schlieren mode, only the ray perpendicular to the background image can be captured with a camera. This is helpful to measure wavefront aberrations that occur after a planar wavefront has passed through the flow-field. Based on these characteristics of BOS of Schlieren mode, a new wavefront measurement technique, which is called the BOS-based wavefront technique (BOS-WT), is proposed in this paper. It works by constructing the relationship between the displacement of the background image and the aero-optical wavefront gradient and uses the Southwell wavefront reconstruction algorithm. A BOS-WT system was assembled, and its temporal resolution was found to be $6 \mathrm{~ns}$, and its temporal-correlation resolution reached $0.2 \mu \mathrm{s}$. A BOS-WT can measure the time-correlation transient wavefront quantitatively. It is simple and easy to operate. In this paper, we also present a study of the aero-optical performance of supersonic mixing layer based on our BOS-WT transient wavefronts at an interval of 5 $\mu \mathrm{s}$. The results showed the wavefront was transient and distorted after it had passed through the mixing layer. Through the analysis of the data at the $5 \mu$ s interval, the temporal evolution of wavefront can be obtained.
\end{abstract}

BOS, aero-optics, wavefront

Citation: Tian L F, Yi S H, Zhao Y X, et al. Aero-optical wavefront measurement technique based on BOS and its applications. Chinese Sci Bull, 2011, 56: 23202326, doi: 10.1007/s11434-011-4583-Z

When a target is imaged through a flow-field with index-ofrefraction variations, the refraction of the light beam will lead to an offset relative to its real location. This offset depends strongly on the distribution of the index-of-refraction. Based on this principle and the Gladstone-Dale equation, Meier [1] developed the BOS technique in 1998 to measure the density of a compressible flow-field quantitatively. In a BOS system, a background image and a charge-coupled device (CCD) are positioned on opposite sides of the flowfield. The background images captured by the CCD without and with disturbance from the flow-field are called the reference image and the experiment image. The displacement

*Corresponding author (email: zhaixing_nomin@ @sohu.com) between them can be computed using cross correlation algorithms. Compared with traditional Schlieren, the facility of a BOS system is simpler. Also, BOS can measure the density gradients quantitatively in both the directions that are perpendicular to the light beam. Since its first demonstration, many researchers have been studying and estimating the properties of BOS, including its sensitivity, accuracy and spatial resolution. This has shown that the accuracy of BOS is very high [2-4]. However, there is some difficulty in studying the detached shock wave [3]. The sensitivity of a BOS system is primarily dependent on the arrangement of the BOS setup, the resolution of the CCD and the focal length of the CCD lens [5]. For BOS, the spatial resolution depends on the integration window size, grid size, and 
magnification factor [2]. Similar to traditional Schlieren, BOS is usually used for planar or axisymmetric flow-fields [2-4,6,7]. For three-dimensional steady flows, a BOS system performs several measurements in various directions, and then reconstructs the results. For unsteady flows, however, several cameras and background images must be used to perform simultaneous measurements [5]. Additionally, the surrounding landscape can be used in BOS background images for full-scale flight tests [8-11].

As mentioned above, aero-optical theory is the underlying principle of BOS. Zhao et al. used a BOS system of PIV (particle image velocimetry) mode to study the aero-optical aberration and jitter imposed by a supersonic mixing layer [12]. However, the distorted wavefront was not measured quantitatively. In this paper, we propose a new wavefront measurement technique called BOS-WT. BOS-WT uses aero-optical theory and a BOS system of Schlieren mode to obtain the relationship between background displacement and wavefront gradient. Moreover, it adopts the Southwell algorithm to reconstruct a distorted wavefront. BOS-WT can measure transient wavefront quantitatively, has high temporal resolution, high temporal correlation resolution and relatively high spatial resolution. The BOS-WT system is simple and easy to operate. In addition, the aero-optical performance of a supersonic mixing layer was studied in this paper using BOS-WT.

\section{BOS-WT}

A typical BOS setup has two modes: PIV mode and Schlieren mode, which are shown in Figure 1. The setup of PIV mode is simpler than that of the Schlieren mode, and is more usable in full-scale flight tests, such as the flow-fields around helicopter airscrews [9], explosions [8] and gunshots flow-field [10]. The relatively low spatial resolution is the result of a lack of BOS of PIV mode. This arises because most of the light rays received by the CCD have a large angle of divergence after transmission through the large flow region. Compared with the PIV mode, Schlieren mode BOS is more complex, because it uses a convex lens and diaphragm. The convex lens and CCD have the same optical axis, and the diaphragm is positioned in the focal plane of the convex lens. Schlieren mode BOS has higher spatial resolution, because only the rays parallel with the optical axis of the convex lens can pass through the diaphragm. From Figure 1, it can be seen that the measured flow-field cannot extend beyond the aperture of the convex lens. Therefore, Schlieren mode BOS is more applicable in laboratory and wind tunnel base experiments.

\subsection{Principle of BOS-WT}

In a BOS setup of Schlieren mode, only rays parallel with the optical axis of the camera lens can pass through the diaphragm and be captured by the camera. This is advantageous for the study of wavefront aberrations induced by a flow-field. Because of this, we can construct the relationship between the background image displacement and the gradient of wavefront aberration. We then adopt a wavefront reconstruction algorithm to compute the wavefront aberration.

As shown in Figure 2, $H$ is the distance between background image and the flow-field center, and $W$ is the width of the flow-field. $\alpha_{y}$ is the deflection angle of the parallel rays from point $A . \Delta y_{A}$ and $\Delta y_{B}$ are virtual offsets of points $A$ and $B . \mathrm{S}$ is the distorted wavefront after it has passed through the flow-field. According to the Malus law, the angle between $\mathrm{S}$ and $y$ is equal to the deflection angle $\alpha_{y}$ of the local rays, as shown in Figure 2.

In the case $W<<2 H$, we obtain the following equations according to the geometrical relationships shown in Figure 2:

$$
\left\{\begin{array}{l}
\alpha_{x}=\frac{\Delta x}{H}, \\
\alpha_{y}=\frac{\Delta y}{H} .
\end{array}\right.
$$

Optical path length (OPL) variation is a typical characteristic used to describe optical aberration. Two-dimensional OPL is defined by

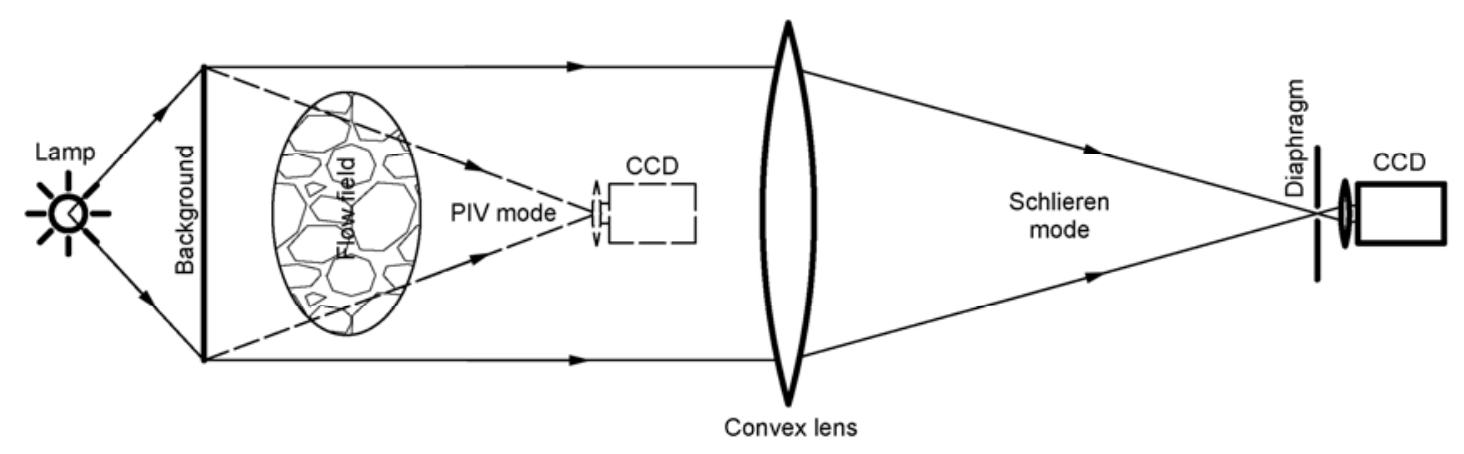

Figure 1 Two BOS setup modes: PIV mode and Schlieren mode. 


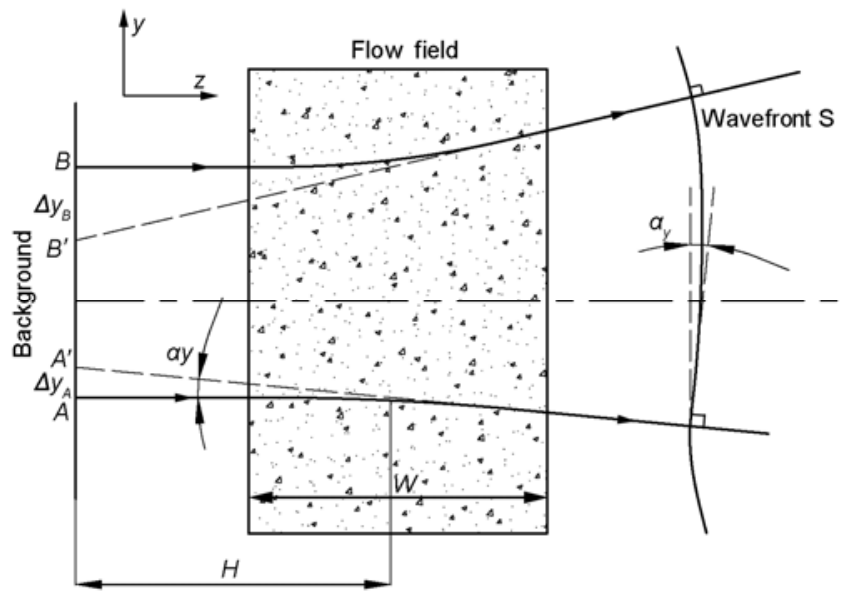

Figure 2 Beam path of a BOS system of Schlieren mode.

$$
O P L(x, y)=\int_{z_{1}}^{z_{2}} n(x, y, z) \mathrm{d} z
$$

Using the Malus law and Figure 2, the gradient of the OPL is

$$
\left\{\begin{array}{l}
\frac{\partial O P L(x, y)}{\partial x}=-\alpha_{x}, \\
\frac{\partial O P L(x, y)}{\partial y}=-\alpha_{y} .
\end{array}\right.
$$

In practice, optical path difference (OPD) is more important than measuring an absolute OPL. OPD is defined as

$$
O P D(x, y)=O P L(x, y)-\overline{O P L(x, y)}
$$

where the overbar denotes a spatial average over the aperture. There are several methods to solve eqs. (1) and (3),

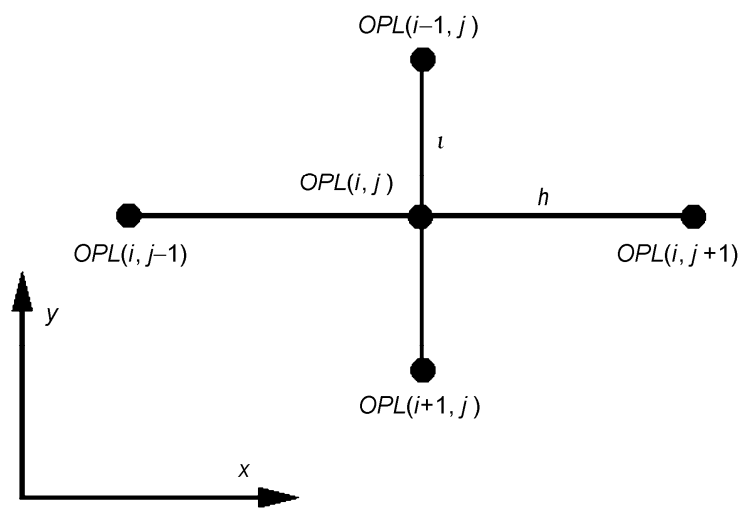

Figure 3 Diagram of the grid.

such as trapezoidal integration, polynomial fitting and the Southwell algorithm [13].

The Southwell algorithm, which is iterative, was adopted to construct the wavefront in this paper. For point $(i, j)$, the wavefront $\operatorname{OPL}(i, j)$ can be integrated from the four neighboring points, as shown in Figure 3. The final value is the weighted average of the four values:

$$
\begin{aligned}
& O P L(i, j)=O P L(i, j-1)-\frac{h}{2 H}\left[\Delta x^{\prime}(i, j-1)+\Delta x^{\prime}(i, j)\right] \\
& O P L(i, j)=O P L(i, j+1)+\frac{h}{2 H}\left[\Delta x^{\prime}(i, j+1)+\Delta x^{\prime}(i, j)\right] \\
& O P L(i, j)=O P L(i-1, j)+\frac{l}{2 H}\left[\Delta y^{\prime}(i-1, j)+\Delta y^{\prime}(i, j)\right] \\
& O P L(i, j)=O P L(i+1, j)-\frac{l}{2 H}\left[\Delta y^{\prime}(i+1, j)+\Delta y^{\prime}(i, j)\right] .
\end{aligned}
$$

The weighted average of the four values is

$$
\begin{aligned}
O P L(i, j) & =\frac{\omega_{i, j-1} O P L(i, j-1)+\omega_{i, j+1} O P L(i, j+1)+\omega_{i-1, j} O P L(i-1, j)+\omega_{i+1, j} O P L(i+1, j)}{\omega_{i, j-1}+\omega_{i, j+1}+\omega_{i-1, j}+\omega_{i+1, j}} \\
& +\frac{\left\{\begin{array}{l}
-\frac{h}{2 H} \omega_{i, j-1}\left[\Delta x^{\prime}(i, j-1)+\Delta x^{\prime}(i, j)\right]+\frac{h}{2 H} \omega_{i, j+1}\left[\Delta x^{\prime}(i, j+1)+\Delta x^{\prime}(i, j)\right] \\
+\frac{l}{2 H} \omega_{i-1, j}\left[\Delta y^{\prime}(i-1, j)+\Delta y^{\prime}(i, j)\right]-\frac{l}{2 H} \omega_{i+1, j}\left[\Delta y^{\prime}(i+1, j)+\Delta y^{\prime}(i, j)\right]
\end{array}\right.}{\omega_{i, j-1}+\omega_{i, j+1}+\omega_{i-1, j}+\omega_{i+1, j}} .
\end{aligned}
$$

The weight factors of the points inside and outside of the aperture are 1 and 0 . After all the points had been covered, a second iteration is made. The iteration number can be based on a user specified value or on the difference between adjacent iterations. The Southwell algorithm is not sensitive to boundary conditions. When the boundary condition differs from the real value, a good result can still be achieved if the iteration number is large enough. However, if the boundary condition and real value are well-matched, merely a few iterations could yield a good result. This reduces the computation time. After the $\operatorname{OPL}(i, j)$ is found, $\operatorname{OPD}(i, j)$ can be calculated using the eq. (4).
BOS-WT measures the deflection angle of a collimated light beam using the BOS technique. Therefore, its accuracy and sensitivity depend strongly on the characteristics of the BOS system. Sourgen et al. [2,3] and Elsinga et al. [4] studied the accuracy via comparison of experimental data derived using BOS, differential interferometry and computational fluid dynamics. The results showed that the accuracy of BOS is about $2 \%$ in most regions of the flow field. This accuracy is sufficient such that this technique could be applied to most flow-fields. However, the accuracy of BOS along a conical shock and detached shock wave is about $7.5 \%$. Goldhahn and Seume's study [5] on the sensitivity of 
BOS showed that the sensitivity primarily depends on the focal length of the lens used, the relative position of the object between camera and background, and the smallest shift in the image plane that can be detected. The deflection angle of the light beam $\varepsilon$ can be computed by

$$
\varepsilon \approx(1+m / l) \cdot v_{p x}\left(\frac{g-f}{g \times f}\right) \cdot \cos ^{2}(\beta),
$$

where $m$ is the distance between camera and the center of the flow-field; $l$ is the distance between the background image and the center of the flow-field; $v_{p x}$ is the pixel shift on the sensor chip of the camera; $g$ is the distance between the camera and the background image; $f$ is the focal length of the camera; and $\beta$ is the angle between the optical axis of the camera lens and a line connecting the center of the lens aperture and a given point in the background image.

\subsection{BOS-WT system}

A schematic of the BOS-WT system used in this paper is shown in Figure 4. This system uses a dual-cavity Nd:YAG laser, which has a pulse width of $6 \mathrm{~ns}$, peak pulse energy of $500 \mathrm{~mJ}$, and a wavelength centered at $532 \mathrm{~nm}$, as its light source with. A high-resolution double-exposure CCD was used in this system. The shortest time interval for doubleexposure of the CCD is $0.2 \mu \mathrm{s}$, and the resolution of the CCD is $2000 \times 2000$ pixels. The CCD and laser are controlled using a synchronizer to ensure that the two laser beams coincide with the frames of the double-exposure respectively. The temporal accuracy of the synchronizer is 250 ps. The performance of the BOS-WT setup is determined by the characteristics of the components of the system. The temporal resolution of this BOS-WT system is 6 $\mathrm{ns}$, and its temporal-correlation resolution is $200 \mathrm{~ns}$. Also, its spatial resolution reaches the millimeter scale.

\section{Application of BOS-WT in supersonic mixing layer flow-field}

Supersonic mixing layers are important for scramjets [14], aero-optics [15] and high-energy-laser weapon systems

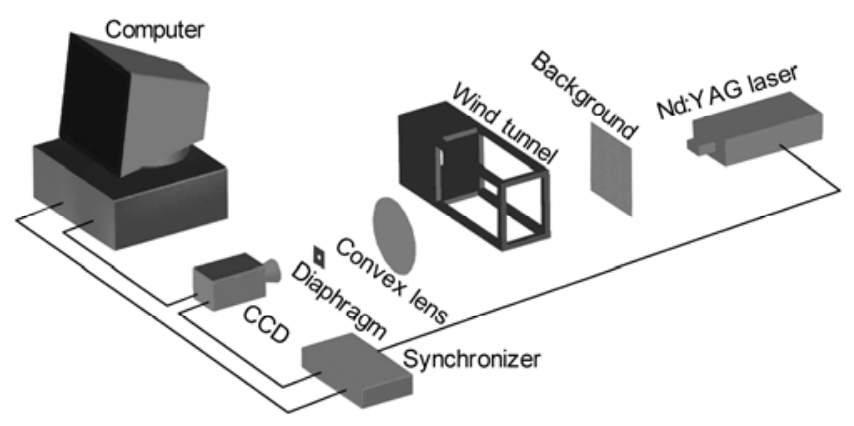

Figure 4 BOS-WT setup.
[16]. For missiles with optical seeking systems, the study of aero-optical effects induced by supersonic mixing layer is very important for guidance accuracy. For this study, an air-breathing supersonic mixing layer wind tunnel was used, whose total pressure $P_{0}=1 \mathrm{~atm}$ and total temperature $T_{0} \approx$ $300 \mathrm{~K}$. The Mach numbers of the upper and lower layers were 2.6 and 3.6. This is shown in Figure 5. Moreover, the background image and CCD are positioned on opposite sides of the wind tunnel. The distance $(H)$ between the center of test section and the background image is $440 \mathrm{~mm}$. Reference image was captured when there was no flow-field in the test section, and the two experiment images were captured when there was disturbance from flow-field at interval of $5 \mu \mathrm{s}$. The displacement profile between the reference and experiment images were calculated using a cross correlation.

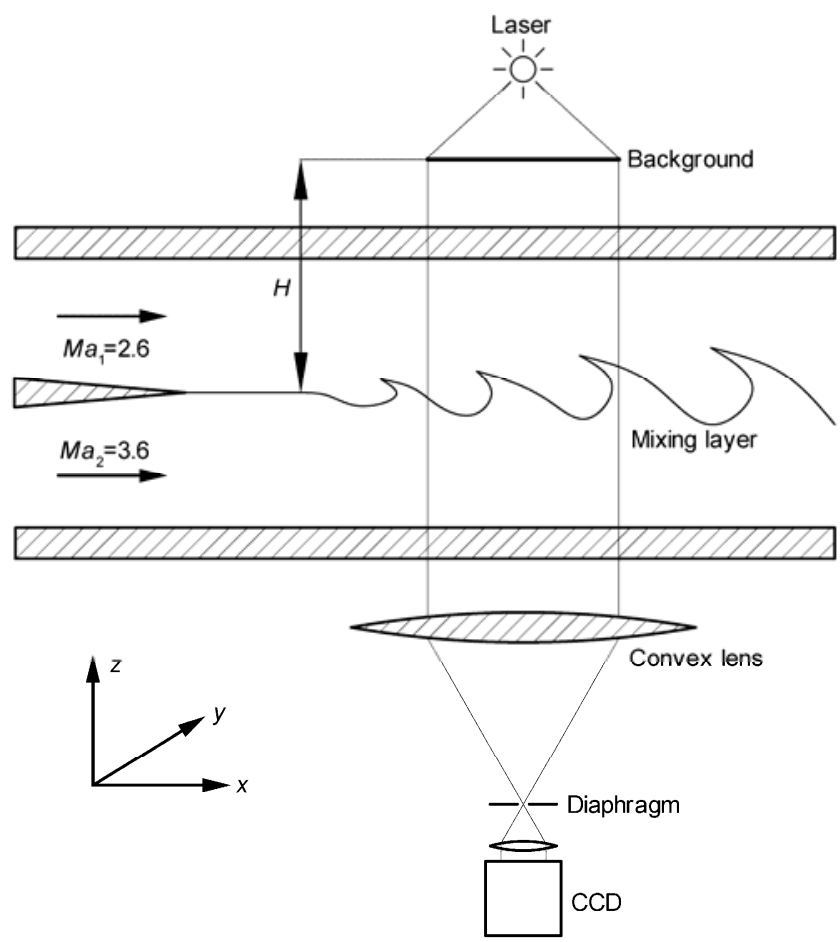

Figure 5 BOS-WT system for the supersonic mixing layer.

There are relative displacements between the reference and experimental images, which are affected by the supersonic mixing layer. The displacements can be calculated using the algorithms used for PIV (particle image velocimetry). This is shown in Figure 6.

The displacement fields between the reference image and experiment images for an interval of $5 \mu$ s are shown in Figure 7. These displacement fields consist of the displacements in both the $x$ and $y$ directions, which is an advantage over traditional Schlieren systems. The size of the integration windows is $0.48 \mathrm{~mm} \times 0.48 \mathrm{~mm}$, which is also the spatial resolution of the displacement fields. The coordinates in 


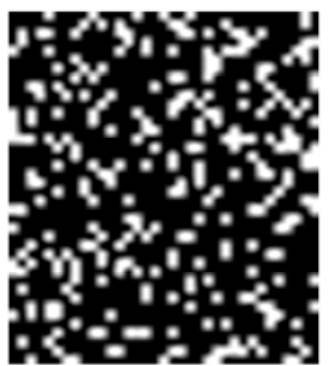

(a) Reference image

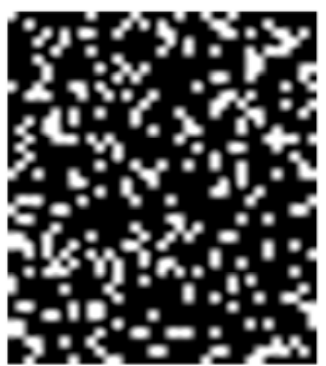

(b) Experiment image

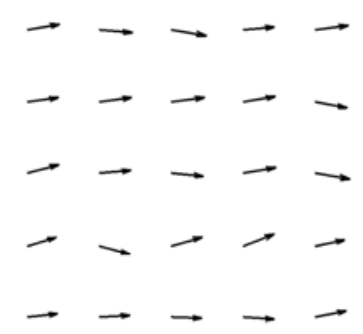

(c) Displacement field

Figure 6 Displacement field computation.

Figure 7 were normalized using the length of the flow-field in the $x$ direction, and the unit used is a pixel. The ribbon- like structure of the displacement can be seen in Figure 7(a) and (d). This is imposed by the coherent structures of

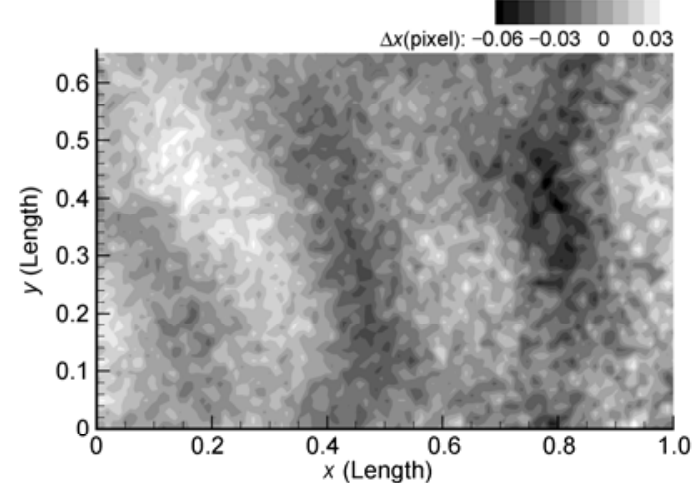

(a) $\Delta x$ contour $(t=0 \mu \mathrm{s})$

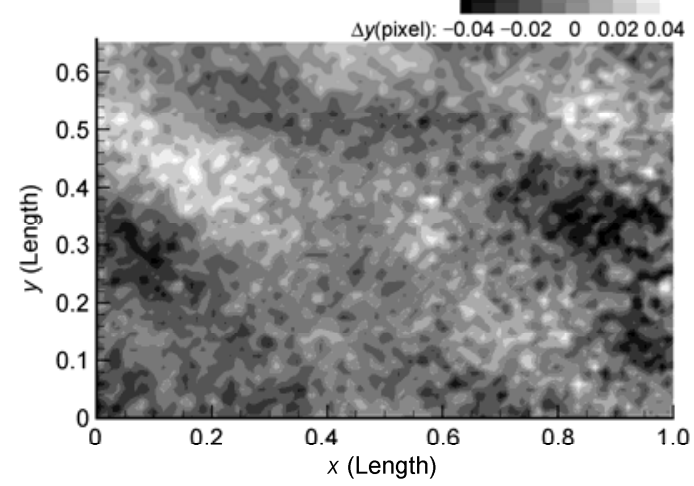

(b) $\Delta y$ contour $(t=0 \mu \mathrm{s})$

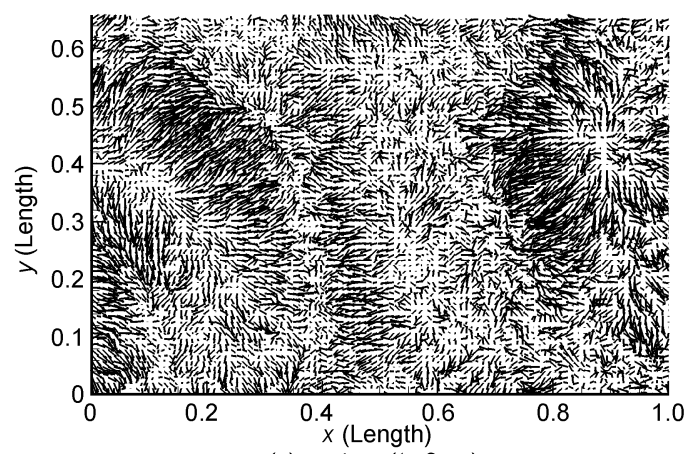

(c) vectors $(t=0 \mu \mathrm{s})$

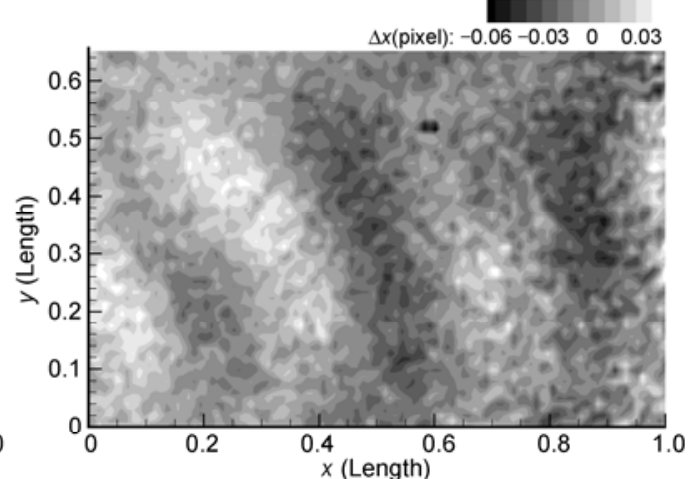

(d) $\Delta x$ contour $(t=5 \mu \mathrm{s})$

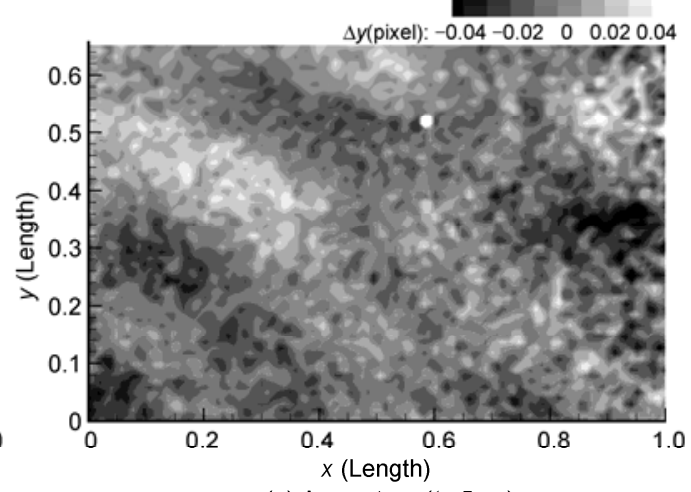

(e) $\Delta y$ contour $(t=5 \mu \mathrm{s})$

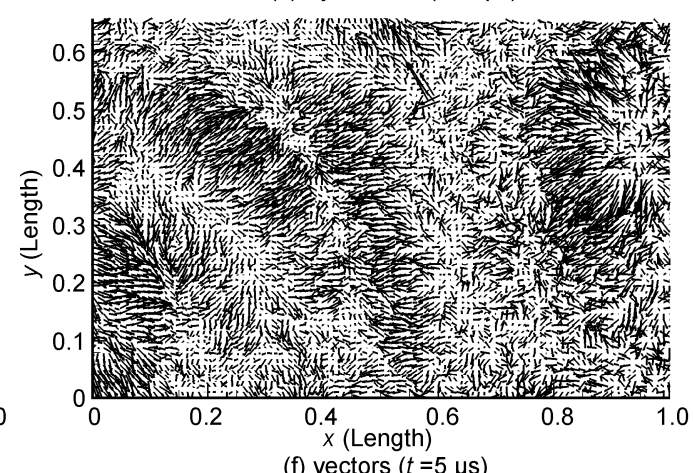

(f) vectors $(t=5 \mu \mathrm{s})$

Figure 7 Displacement field of background image. (a), (b) and (c) are displacement contours in $x$ and $y$ directions, and the displacement vectors, respectively, at $t=0 \mu \mathrm{s}$. (d), (e) and (f) are displacement contours in the $x$ and $y$ directions, and the displacement vectors, respectively, at $t=5 \mu$ s. 
the supersonic mixing layer [17]. A comparison of the displacement fields at interval of $5 \mu$ s shows that the displacement field moves downstream. However its induced deformation cannot be seen clearly.

Substituting the results shown in Figure 7 into eqs. (1) and (3), the Southwell algorithm will allow for the reconstruction the wavefront aberration created by supersonic mixing layer. This is shown in Figure 8. The variation in the wavefront is not large, and the variation in the $x$ direction is larger than that in the $y$ direction. It appears that there is a periodicity to the wavefront in the $x$ direction that matches the periodicity of the displacement field. However, there is no such behavior in the wavefront in the $y$ direction. A comparison of the wavefront at different time shows that the wavefront moves downstream, and its associated deformation is not largely apparent after $5 \mu \mathrm{s}$.
To estimate the validity and accuracy of the BOS-WT, the wavefronts measured using BOS-WT were compared with the wavefronts measured using NPLS-WT (NPLSbased wavefront technique) [18]. NPLS-WT can be used to measure wavefront aberration induced by a section of a flow field at high spatiotemporal resolution, and can avoid the influence from environmental disturbances. The wavefronts induced by the supersonic mixing layer, which are shown in Figure 5 for BOS-WT measurements, were measured using NPLS-WT. The results are shown in Figure 9. A comparison between Figures 8 and 9 shows that the wavefronts measured using BOS-WT and NPLS-WT have the same dimensions, and the difference between them is relatively small. The reason for the small difference between them is that BOS-WT is affected by the environmental disturbances and NPLS-WT is not.
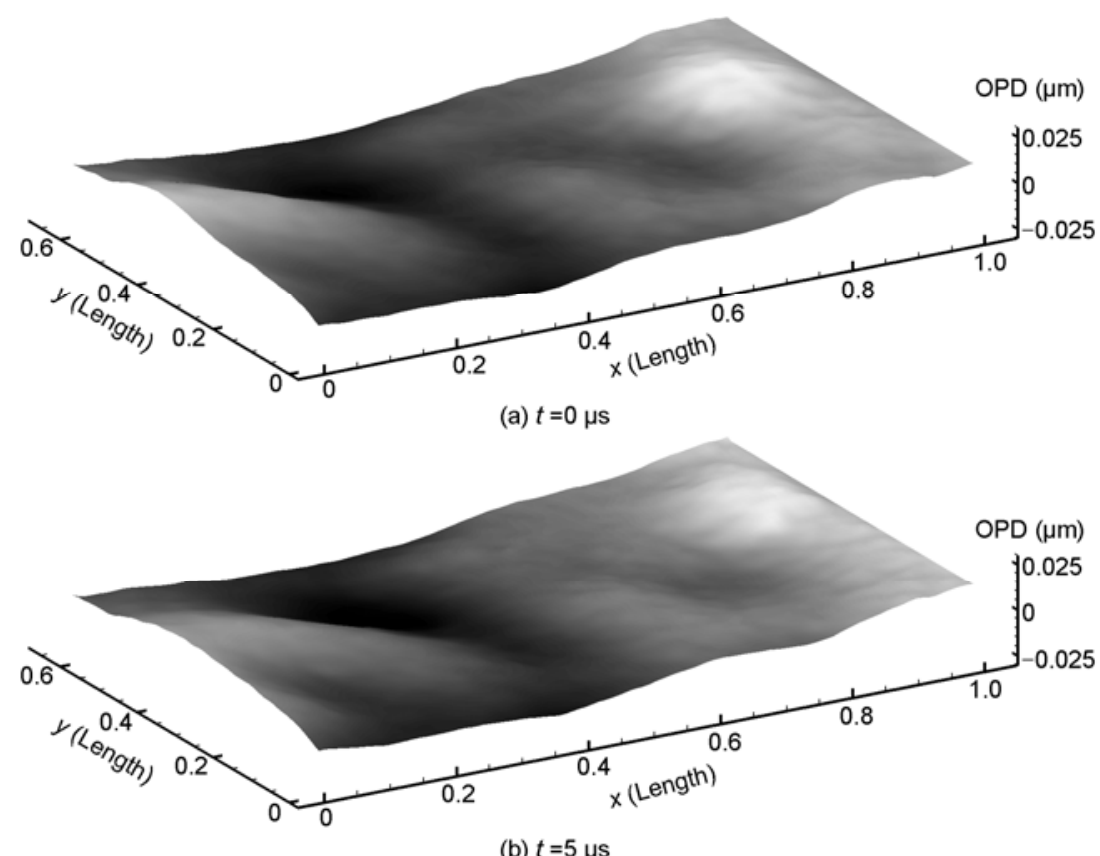

Figure 8 Reconstructed wavefront aberration from the data in Figure 7.

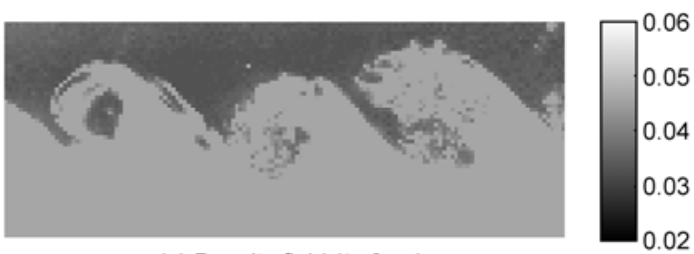

(a) Density field $(t=0 \mu \mathrm{s})$

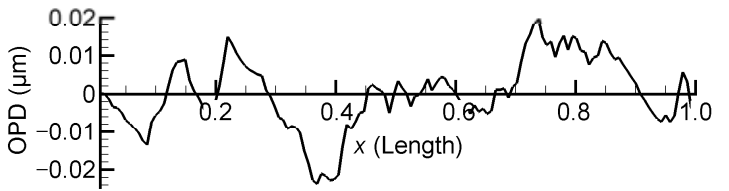

(c) OPD $(0 \mu \mathrm{s})$

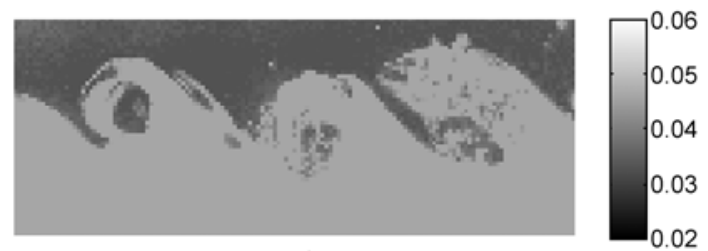

(b) Density field $(t=5 \mu \mathrm{s})$

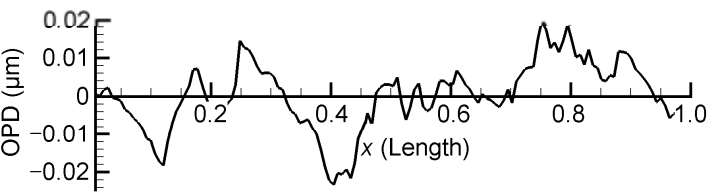

(d) OPD $(5 \mu \mathrm{s})$

Figure 9 Wavefront aberration induced by supersonic mixing layer measured using NPLS-WT. (a) and (b) are density fields of the mixing layer at an interval of $5 \mu$ s. (c) and (d) are wavefront aberrations induced by the flow field shown in (a) and (b). 


\section{Conclusions}

Based on the aero-optical principle of BOS technique, a new wavefront measurement technique, called BOS-WT, is proposed in this paper. It works by constructing the relationship between the displacement from the background image and the gradient of the aero-optical wavefront. The BOS-WT can measure transient wavefronts quantitatively, and its facility is very simple and can be operated easily. The temporal resolution of the BOS-WT system demonstrated in this study is $6 \mathrm{~ns}$, and its temporal correlation resolution can reach up to 200 ns. In this study, the aero-optical performance of a supersonic mixing layer was studied by BOS-WT, and transient wavefronts were measured. The results revealed the transient distorted wavefront. By analyzing the results over an interval of $5 \mu \mathrm{s}$, we found that the wavefront moves downstream, but the shape of the deformation cannot be seen clearly.

1 Meier G E A. New optical tools for fluid mechanics. In: Proceedings of the 8th International Symposium on Flow Visualization, Sorrento, Italy, 1998

2 Sourgen F, Haertig J, George A, et al. Validation of CFD density field in supersonic axisymmetric flow using BOS and differential interferometry. AIAA Paper, 2005, 2005-6036

3 Sourgen F, Haertig J, Rey C. Comparison between background oriented schlieren measurements (B.O.S.) and numerical simulations. AIAA Paper, 2004, 2004-2602

4 Elsinga G E, Van Oudheusden B W, Scarano F, et al. Assessment and application of quantitative schlieren methods: Calibrated color schlieren and background oriented schlieren. Exp Fluids, 2004, 36:
$309-325$

5 Goldhahn E, Seume J. The background oriented schlieren technique: Sensitivity, accuracy, resolution and application to a three-dimensional density field. Exp Fluids, 2007, 43: 241-249

6 Venkatakrishnan L, Meier G E A. Density measurements using the background oriented schlieren technique. Exp Fluids, 2004, 37: 237-247

7 Meier G E A. Computerized background-oriented schlieren. Exp Fluids, 2002, 33: 181-187

8 Sommersel O K, Bjerketvedt D, Christensen S O, et al. Application of background oriented schlieren for quantitative measurements of shock waves from explosions. Shock Waves, 2008, 18: 291-297

9 Raffel M, Richard H, Meier G E A. On the applicability of background oriented optical tomography for large scale aerodynamic investigations. Exp Fluids, 2000, 28: 477-481

10 Hargather M J, Settles G S. Natural-background-oriented schlieren imaging. Exp Fluids, 2010, 48: 59-68

11 Kindler K, Goldhahn E, Leopold F, et al. Recent developments in background oriented schlieren methods for rotor blade tip vortex measurements. Exp Fluids, 2007, 43: 233-240

12 Zhao Y X, Yi S H, Tian L F, et al. An experimental study of aero-optical aberration and dithering of supersonic mixing layer via BOS. Sci China: Phys Mech Astron, 2010, 53: 81-94

13 Southwell W H. Wave-front estimation from wave-front slope measurements. J Opt Soc Am, 1980, 70: 998-1006

14 Wakamatsu Y, Kanda T, Yatsuyanagi N. Preliminary consideration of hypersonic test vehicle for scramjet engine test. ISTS 2000-g-24, 2000

15 Chew L. Coherent structures effects on shear layer optics. AIAA Paper, 1990, 90-0185

16 Yi S H, Hou Z X, Zhao Y X. Supersonic Free-Vortex Aerowindow and Its Optical Quality (in Chinese). Changsha: NUDT Press, 2005

17 Yi S H, He L, Zhao Y X, et al. A flow control study of a supersonic mixing layer via NPLS. Sci China Ser G: Phys Mech Astron, 2009, 52: 2001-2006

18 Yi S H, Tian L F, Zhao Y X, et al. Aero-optical aberration measuring method based on NPLS and its application. Chinese Sci Bull, 2010, 55: 3545-3549

Open Access This article is distributed under the terms of the Creative Commons Attribution License which permits any use, distribution, and reproduction in any medium, provided the original author(s) and source are credited. 